\title{
Regulation of follistatin-like 3 expression by miR-486-5p modulates gastric cancer cell proliferation, migration and tumor progression
}

\author{
Zhou-Tong Dai ${ }^{1,{ }^{*}}$, Yuan Xiang ${ }^{1,2,{ }^{*}}$, Xiao-Yu Zhang ${ }^{1,{ }^{*}}$, Qi-Bei Zong ${ }^{1}$, Qi-Fang $\mathrm{Wu}^{1}$, You Huang ${ }^{1}$, \\ Chao Shen ${ }^{1}$, Jia-Peng $\mathrm{Li}^{1}$, Sreenivasan Ponnambalam ${ }^{3}$, Xing-Hua Liao ${ }^{1}$ \\ ${ }^{1}$ Institute of Biology and Medicine, College of Life and Health Sciences, Wuhan University of Science and \\ Technology, Hubei 430081, P.R. China \\ ${ }^{2}$ Department of Medical Laboratory, Central Hospital of Wuhan, Tongji Medical College, Huazhong University of \\ Science and Technology, Wuhan 430014, P.R. China \\ ${ }^{3}$ School of Molecular and Cellular Biology, University of Leeds, Leeds LS2 9JT, United Kingdom \\ *Equal contribution
}

Correspondence to: Sreenivasan Ponnambalam, Xing-Hua Liao; email: s.ponnambalam@leeds.ac.uk; xinghualiao@hotmail.com, https://orcid.org/0000-0002-8067-4851

Keywords: follistatin, FSTL3, gastric cancer, miR-486-5p, cell proliferation

Received: March 11, $2021 \quad$ Accepted: August 2, $2021 \quad$ Published: August 23, 2021

Copyright: (C) 2021 Dai et al. This is an open access article distributed under the terms of the Creative Commons Attribution License (CC BY 3.0), which permits unrestricted use, distribution, and reproduction in any medium, provided the original author and source are credited.

\section{ABSTRACT}

Cancer development and progression can be regulated by the levels of endogenous factors. Gastric cancer is an aggressive disease state with poor patient prognosis, needing the development of new diagnostics and therapeutic strategies. We investigated the close association between follistatin-like 3 (FSTL3) and different cancers, and focused on its role in gastric cancer cell function. Using cancer bioinformatics, we found that FSTL3 expression is elevated in a large majority of the $\mathbf{3 3}$ cancers we analyzed in publicly available cancer databases. Elevated levels of FSTL3 is associated with poor patient prognosis in gastric cancer. In a comparison of normal gastric epithelial cells and gastric cancer cell lines, FSTL3 expression was consistently elevated in gastric cancer cells. Overexpression of FSTL3 promoted gastric cancer cell viability, proliferation and migration. Conversely, FSTL3 knockdown inhibits these cellular processes. Using bioinformatics, we found that the FSTL3 mRNA has a potential binding site in the $3^{\prime}$-UTR for a small microRNA, miR-486-5p. Further bioinformatics revealed significant negative correlation between FSTL3 and miR-486-5p levels. Using luciferase reporter constructs, we provide evidence that the 3'UTR from the FSTL3 mRNA can confer downregulation in the presence of miR-486$5 p$. These studies lead us to conclude that FSTL3 has oncogenic properties and increased expression of this gene product promotes gastric cancer development and progression.

\section{INTRODUCTION}

Globally, gastric cancer is one of the most common malignant cancers with serious consequences for population health and mortality. The 2020 Cancer Statistics Survey shows that the gastric cancer mortality rate is $\sim 40 \%$ [1]. The development and use of gastroscopy has dramatically increased diagnosis and treatment, this is most effective in early stage disease
[2-4], whereas most gastric cancer patients present in the latter stages of disease to the clinic. Gastric cancer is associated with significant secondary metastases which cannot be treated by conventional surgery [5]. The prognosis of advanced stage gastric cancer patients is low, with a typical survival period of 8-12 months [68]. Gastric cancer tumor infiltration and metastasis are key causes of poor patient prognosis [9]. Mechanistic explanations of such effects include oncogene 
activation, tumor suppressor inactivation, and abnormal cellular metabolism. The emergence of cancer bioinformatics has led to the rapid identification and validation of new cancer markers and prognostic indicators. For example, Pan and colleagues found that layilin (LAYN) is a new prognostic and immune infiltration marker in gastric cancer [10]. Aberrant expression of the homeobox-related transcription factor HOXC10 is also linked to gastric cancer cell migration, proliferation and tumor development [11]. The application of cancer bioinformatics is thus a powerful tool in identifying new gastric cancer biomarkers and therapeutic targets.

The follistatin family of secreted glycoproteins includes follistatin-like 3 (FSTL3). More than 20 yrs ago, it was discovered that the FSTL3 locus undergoes chromosomal rearrangement linked to development of non-Hodgkin lymphoma [12]. Subsequent work has implicated FSTL3 in cellular differentiation, insulin resistance, aging, obesity and arteriosclerosis [13-16]. However, recent studies implicated FSTL3 in the regulation of a range of cancers. For example, FSTL3 expression can be used to distinguish between benign and malignant breast cancer states [17]. Moreover, an FSTL3 antagonist inhibited breast cancer cell proliferation [18]. However, how FSTL3 regulates cancer development and progression is unclear; furthermore, very little is known about FSTL3 regulation of gastric cancer.

Non-coding RNAs (ncRNAs) usually do not encode protein but regulate protein translation and/or mRNA degradation [19]. Such ncRNAs include lncRNAs, microRNAs and circRNAs [20]. A microRNA (miRNA) is an endogenously expressed non-coding RNA 18-25 nucleotides in length, which frequently bind to the 3'-untranslated region (3'-UTR) of the target mRNA, causing degradation or a block in translation by ribosomes [21]. Abnormal miRNA expression can affect cancer development and progression by modulating cell proliferation, migration and invasion $[22,23]$. The identification of new gastric cancer biomarkers associated with such features of cancer cell behavior could provide new opportunities for disease intervention.

In this study, we used cancer bioinformatics to evaluate FSTL3 levels in different cancers in the TCGA database. Further analysis of FSTL3 levels in normal and tumor gastric tissues was analyzed to evaluate the potential role of FSTL3 as a gastric cancer biomarker. We then addressed the role of FSTL3 in gastric cancer using in vitro cellular models alongside in vivo animal models. Our studies now provide new insights into how abnormal control of FSTL3 expression regulates gastric cancer development and progression.

\section{MATERIALS AND METHODS}

\section{Bioinformatics}

All patient data used for this analysis were derived from the public databases such as TCGA [24] (https://tcgadata.nci.nih.gov/tcga/) and GEO (https://www.ncbi.nlm. nih.gov/). $\mathrm{R}$ software was used to analyze gene expression and perform functional gene enrichment. Moreover, using the visualization tools in StarBase [25] (https://web.archive.org/web/20110222111721/http://sta rbase.sysu.edu.cn/), we were able to evaluate the link between miRNA levels and clinical cancer datasets in TCGA. The interaction properties between miRNA and mRNA were predicted using StarBase. Protein-protein interaction (PPI) studies was carried out using the STRING database [26] (https://string-db.org). Cytoscape (https://cytoscape.org/) with the MCODE app was used to analyze the PPI core subnet.

\section{Cell culture}

Human gastric epithelial cell line GES1 and human gastric cancer cell lines BGC-823, MGC-803, SGC7901, AGS, and HGC-27 were purchased from the cell bank at the Chinese Academy of Sciences. MGC-803, SGC-7901, and HGC-27 cells were cultured in RPMI1640, AGS cells cultured in DMEM/F12 and the remaining cell lines cultured in DMEM (GIBCO, USA). All cells were cultured in medium supplemented with $10 \%$ fetal bovine serum (GIBCO, USA), $100 \mu \mathrm{g} / \mathrm{ml}$ penicillin, and $100 \mu \mathrm{g} / \mathrm{ml}$ streptomycin and grown at $37^{\circ} \mathrm{C}$ in $5 \% \mathrm{CO}_{2}$.

\section{Lentiviral transduction}

HEK293T cell line were co-transfected with plasmids pLKO.1, pCDH (synthesized by Shanghai Gema Pharmaceutical Technology Co., Ltd.), VSVG, and GAG-POL using Lipofectamine 3000 (Invitrogen, USA), for the production of replication-defective lentiviral particles. Recombinant lentivirus was harvested at either 48 or $72 \mathrm{~h}$ after plasmid transfection. Cell medium was concentrated using PEG-8000 and virus titer determined. Viral particles were added to cultured cells and stable clones selected using $1 \mu \mathrm{g} / \mathrm{ml}$ puromycin (Sigma-Aldrich, USA), 2-3 days after lentiviral transduction.

\section{Western blotting}

All proteins were separated by SDS-PAGE and then electroblotted onto PVDF membranes (Millipore, USA) 
before blocking with 5\% (w/v) skimmed milk (BD, USA) for $1 \mathrm{~h}$. The blocked PVDF membrane was incubated with diluted primary antibody in blocking buffer at $4^{\circ} \mathrm{C}$ overnight. The next day, the PVDF membrane was rinsed and incubated at room temperature with diluted HRP-conjugated secondary antibodies such as anti-mouse-HRP (Abcam, USA) or anti-rabbit-HRP (Abcam, USA) for 2 h. ECL luminescent solution (Meilunbio, China) was used to visualize bound antibodies using a digital gel imaging system (Biorad, USA). The antibodies used are: mouse anti- $\beta$-Actin used at 1:5000 (CST, USA; \#4970S), FSTL3 used at 1:1000 (Abcam, USA; \#ab232761).

\section{Dual-luciferase reporter assay}

Luc-3'-UTR of FSTL3 and mutant form were separately subcloned into the pmirGLO plasmid (Addgene, USA) to establish wt-FSTL3-luc (WT) and mut-FSTL3-luc (Mut) plasmid constructs respectively. The miRNA mimic, internal control, and parental luciferase plasmid were co-transfected into cells. Luciferase activity was assayed $48 \mathrm{~h}$ after transfection using the DualLuciferase Reporter Assay System (Promega, USA).

\section{RNA isolation and qRT-PCR}

RNeasy Plus Universal Mini Kit (QIAGEN, USA) was used to isolate total RNA from cell lines, and HiScript ${ }^{\circledR}$ II $1^{\text {st }}$ Strand cDNA Synthesis Kit (Vazyme, China) was used to reverse transcribe into cRNA. At the same time, the miRNeasy Micro Kit (QIAGEN, USA) was used to isolate miRNA from cell lines, and the miRNA $1^{\text {st }}$ Strand cDNA Synthesis Kit (Vazyme, China) was used for reverse transcription of miRNA. For the extracted RNA and miRNA, qRT-PCR was performed on the Bio-Rad CFX-96 (Biorad, USA) system using the SYBR Green (Yisen, USA) method to determine the relative RNA levels. $\beta$-actin and U6 mRNAs were used as endogenous controls. The primer sequences used to detect FSTL3 and Ki67 mRNAs are: FSTL3-F: 5-GTGCCTCCGGCAACATTGA-3, FSTL3-R: 5-GCA CGAATCTTTGCAGGGA-3, Ki67-F:5-GGGCCAATC CTGTCGCTTAAT， Ki67-R:5-GTTATGCGCTTGCG AACCT-3. The synthesis of primers, plasmid sequencing, miRNA reverse transcription, sequencing, primer synthesis and FSTL3 siRNA synthesis were done commercially (Ribobio, China).

\section{RNA immunoprecipitation (RIP)}

RNA-protein-antibody complexes were captured using Protein A/G (ThermoFisher, USA). RNA was eluted by adding TRIzol directly to magnetic beads and isolated as per the manufacturer's instructions. cDNA was synthesized using HiScript ${ }^{\circledR}$ II $1^{\text {st }}$ Strand
cDNA Synthesis Kit (Vazyme, China) and analyzed by qRT-PCR.

\section{Cell viability assay}

In a 96-well plate, $3 \times 10^{3}$ cells in a $100 \mu$ l volume were added to each well; each group had 5 multiple wells. After allowing the cells to adhere, $10 \mu \mathrm{l}$ of CCK-8 solution (Dojindo, Japan) was added to each well. The 96-well plate was incubated in an incubator for $1 \mathrm{~h}$, and absorbance measured at $450 \mathrm{~nm}$ using a microplate reader (BioTek, USA). The assay was independently repeated three times.

\section{Cell migration and invasion assay}

A Transwell filter or chamber (Corning, USA) was used to analysis cell migration and invasion. $600 \mu \mathrm{l}$ of cell suspension was added to the upper chamber, and the cells were suspended serum-free medium. Matrigel (BD, USA) was added to the upper chamber for invasion assay or $10 \%$ FBS added to the lower chamber for cell migration assay. $24 \mathrm{~h}$ after incubation, the cells that had migrated through the Transwell membrane filter were fixed with formalin (GBICO, USA) and stained with crystal violet. The number of migrated cells were imaged using an inverted microscope (Olympus, Japan) and fields of cells counted. At least five random fields of view were selected for each calculation, and all determinations were independently repeated three times.

\section{Wounded cell monolayer closure assay}

An in vitro wounded cell monolayer assay was also used to study cell migration. The cells were seeded in 6well plates $\left(1 \times 10^{6}\right.$ per well $)$, and after cell adhesion, linear scratch wounds were made in the cell monolayer using a sterile $200 \mu \mathrm{l}$ plastic pipette tip. Photomicrographs were captured using a digital Olympus camera (Olympus, Japan) $48 \mathrm{~h}$ after treatment or stimulation.

\section{Human-mouse tumor xenograft model}

Animal experiments were conducted under the guidelines of the Laboratory Animal Center of Wuhan University of Science and Technology. BALB/c Nude mice, which were 4 weeks old and $\sim 15 \mathrm{~g}$ weight per mouse were purchased from Beijing Huafukang Experimental Animal Co., Ltd., and housed in the Experimental Animal Center of Wuhan University of Science and Technology. Human gastric cancer cells were digested with trypsin (GIBCO, USA) without phenol red and EDTA, then resuspended in PBS containing 50\% Matrigel (BD, USA). The cell suspension $2 \times 10^{6}$ cells $/ \mathrm{ml}$ was injected subcutaneously 
into the dorsal side of the Nude mice. The mice were sacrificed 4 weeks later for biochemical and histopathological analyses were performed on the tumor samples.

To study metastasis in vivo, $2 \times 10^{5}$ cells of each group were re-suspended in $100 \mu \mathrm{l}$ PBS, and intravenously injected into the tail vein of $\mathrm{BALB} / \mathrm{c}$ nude mice (10 mice/group). After 28 days, the number of mice with metastasis was counted.

\section{Histology and histochemistry}

The tissues were fixed, dehydrated, and paraffinembedded in the $10 \%$ formalin to make $5 \mu \mathrm{m}$ tissue sections for hematoxylin-eosin staining, and observation using a digital microscope (Olympus, Japan). Xylene was used to dewax them twice for 15 min each time. The tissue sections were incubated in $3 \%$ (v/v) $\mathrm{H}_{2} \mathrm{O}_{2}$ (Sigma-Aldrich, St. Louis, MO, USA) at $37^{\circ} \mathrm{C}$ for $30 \mathrm{~min}$, boiling in $0.01 \mathrm{M}$ citric acid buffer at $95^{\circ} \mathrm{C}$ for $20 \mathrm{~min}$, and then blocked with nonspecific serum solution at $37^{\circ} \mathrm{C}$ for $10 \mathrm{~min}$ before incubation with diluted primary antibodies at $37^{\circ} \mathrm{C}$ for $2 \mathrm{~h}$. Tissue sections were then incubated with speciesspecific secondary antibodies conjugated to horseradish peroxidase (HRP) and counterstained with hematoxylin (Meilunbio, China) at room temperature for $4 \mathrm{~min}$. The sections were observed using a digital microscope (Olympus, Japan). The following antibodies were used: rabbit anti-FSTL3 at 1:50 (Abcam, USA; \#ab232761).

\section{Cell adhesion assay}

In a 96-well plate with Matrigel (BD, USA) already spread. 2000 cells were seeded in each well, and serumfree medium was used to cultivate the cells. After $2 \mathrm{~h}$ of incubation, cells adhered to Matrigel were washed twice with PBS. After fixation with $4 \%$ paraformaldehyde (Meilunbio, China) for $10 \mathrm{~min}$, DAPI (Meilunbio, China) staining was used to observe the number of adherent cells under a fluorescence microscope (Olympus, Japan). Each experiment was repeated at least at least 3 times.

\section{Focal adhesion assay}

The experimental method is like immunofluorescence. The following antibodies were used: anti-F-actin (Meilunbio, China), anti-vinculin (CST, USA \#91459).

\section{Statistical analysis}

Comparisons between groups and datasets were performed using $\mathrm{R}$ software version 4.0.3. Comparisons were completed using a one-way analysis of variance (ANOVA) and two-tailed Student's t-test. Correlations were calculated using the Pearson correlation. KaplanMeier analyses were used to analyze cancer patient survival. All data are presented with error bars indicating mean \pm SD. Statistical significance cutoff is at $P<0.05$.

\section{Availability of supporting data}

The data and files generated during this study are available from the corresponding author upon request.

\section{RESULTS}

FSTL3 expression is associated with gastric cancer and poor patient prognosis

FSTL3 expression was profiled in clinical datasets for 33 cancers deposited in the TCGA database (Figure 1A). FSTL3 expression was elevated, notably in CHOL, COAD, GBM, HNSC, KIRC, KIRP, READ, STAD and THCA cancers; however, FSTL3 expression was decreased in $\mathrm{KICH}$, LUAD, LUSC, PCPG, UCEC cancers. To further analyze gastric cancer (stomach adenocarcinoma, STAD) increased FSTL3 expression (Figure 1A) we used the GEO clinical dataset GSE33335. This dataset contains gene profiling data from cancerous tissues and non-cancerous adjacent tissues taken from 25 gastric cancer patients. FSTL3 expression was increased in gastric cancer compared to control tissues (Figure 1B). Next, we evaluated the clinical implications of FSTL3 expression in gastric cancer patient prognosis using Kaplan-Meier analysis (Figure 1C). The median FSTL3 expression in gastric cancer tissues was defined as the cut-off value. All STAD patients were divided into FSTL3 low expression and high expression groups $(\mathrm{n}=175)$. We found that the high FSTL3 expression caused decreased STAD patient survival over a $10 \mathrm{yr}$ period, $P=0.036$ (Figure 1C).

We also performed Univariate Cox analysis to analyze histopathological characteristics, age, gender, and disease staging (Table 1). FSTL3 expression was increased with older age and later stages of gastric cancer disease (Table 1). However, disease grade nor gender had no significant correlation with FSTL3 expression (Table 1). Gastric cancer patients thus exhibit increased FSTL3 expression which influences disease progression and survival.

\section{Gene enrichment and functional analyses for elevated FSTL3 expression}

We screened for differentially expressed genes from the FSTL3 high and low expression groups in gastric cancer 
(Figure 1C). We found 122 differentially expressed genes linked to the STAD database in TCGA and these are depicted as points on the gene expression log plot (Figure 2A). The top 10 differentially expressed genes linked to FSLT3 expression are shown in Figure 2B. These differentially expressed genes analyzed by gene ontology descriptors include extracellular matrix organization, extracellular structure organization, ossification, negative regulation of cellular responses to growth factor stimulus and collagen fibril organization
(Figure 2C). The top five biochemical pathways identified by enrichment analysis using the Kyoto Encyclopedia of Gene and Genomes (KEGG) are focal adhesions, PI3K-Akt signal transduction, ECM-receptor interactions, protein digestion and absorption, and cancer proteoglycans (Figure 2D). These 122 differentially expressed genes were imported into the STRING database to construct PPI networks. These genes can be linked into a singular network with 121 nodes and 173 edges (Figure 2E). COL1A2, COL3A1,
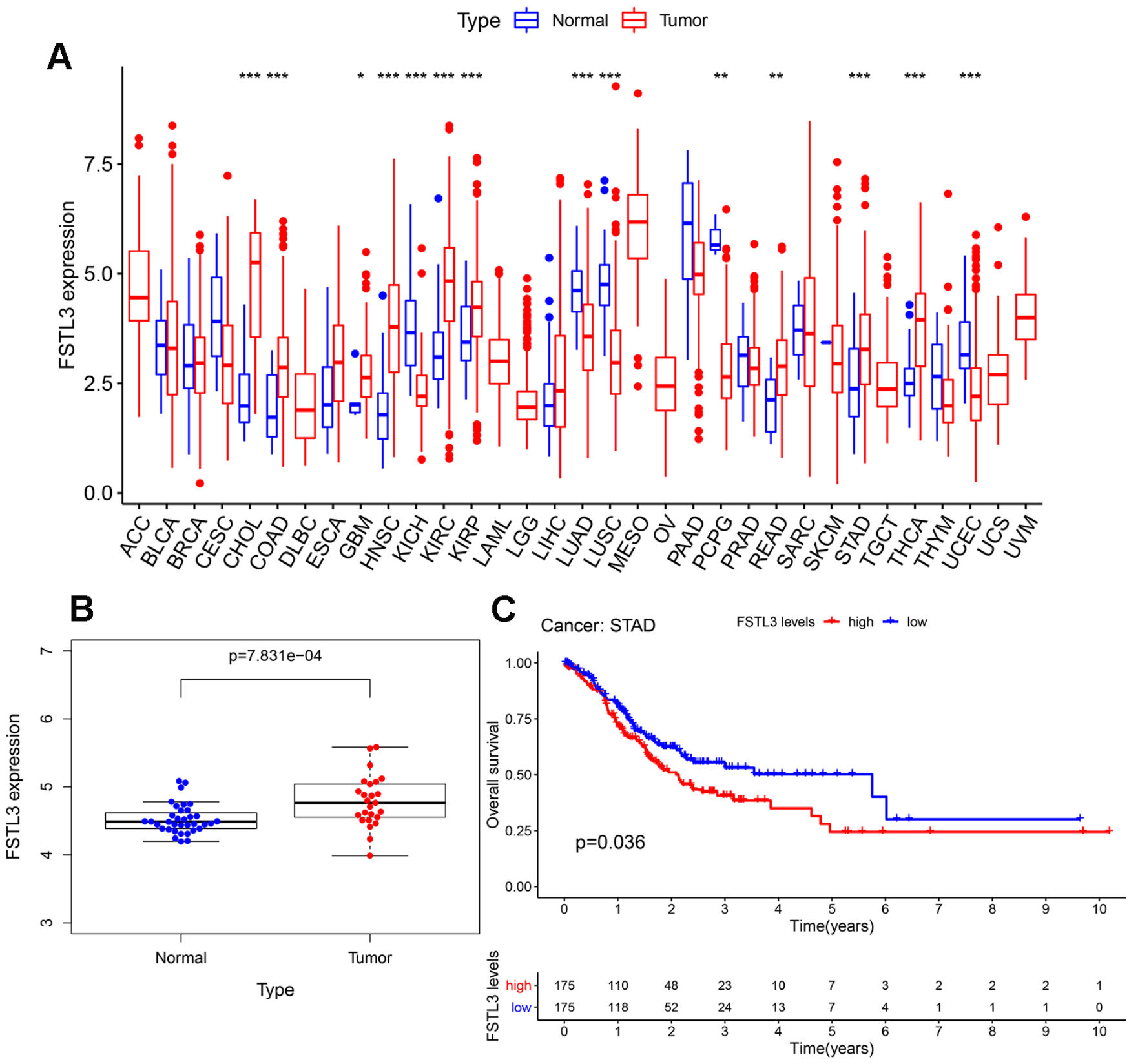

Figure 1. Elevated FSTL3 expression in gastric cancer is linked to poor patient prognosis. (A) Profiling of FSTL3 expression in 33 cancers within the TCGA database. (B) Analysis of FSTL3 expression in non-cancerous and cancerous gastric tissues using the GEO clinical dataset GSE33335. (C) Kaplan-Meier analysis of gastric cancer patient overall survival after classification into high (red) and low (blue) FSTL3 expression groups ( $\mathrm{n}=175)$. Significance indicated as follows: ${ }^{*} P<0.05,{ }^{* *} P<0.01, * * * P<0.001$. 
Table 1. Univariate Cox analysis of gastric cancer patient clinical features linked to increased FSTL3 expression.

\begin{tabular}{lcccc}
\hline & HR & HR.95L & HR.95H & $\boldsymbol{P}$-value \\
\hline age & 1.026965748 & 1.007829689 & 1.046465151 & $\mathbf{0 . 0 0 5 5 6 0 1 7}$ \\
gender & 1.483828302 & 0.979779065 & 2.247186647 & 0.062392212 \\
grade & 1.367875492 & 0.946580494 & 1.976676441 & 0.095375967 \\
stage & 1.535478376 & 1.221185829 & 1.930659353 & $\mathbf{0 . 0 0 0 2 4 2 4 9 7}$ \\
T & 1.297541887 & 1.023344886 & 1.645207762 & $\mathbf{0 . 0 3 1 5 1 6 7 1 1}$ \\
M & 2.048306027 & 1.096196896 & 3.827375896 & $\mathbf{0 . 0 2 4 5 8 1 6 9 3}$ \\
$\mathbf{N}$ & 1.267206892 & 1.068895108 & 1.5023114 & $\mathbf{0 . 0 0 6 3 8 6 5 2 8}$ \\
\hline
\end{tabular}

Bold values are statistically significant $(P<0.05)$.

COL1A1, COL5A1, COL5A1 constitute the top five nodes. By using the MYOCD tool in Cytoscape, we identified core subnet modules within this PPI network; the top three nodes are depicted (Figure 2F).

\section{Elevated FSTL3 levels promote gastric cell proliferation and migration}

The expression of FSTL3 in a range of gastric cell lines was evaluated (Figure 3). Normal gastric epithelial cell line GES1 was compared to 5 different gastric cancer cell lines and Western blotting of cell lysates showed clear differences in FSTL3 protein levels (Figure 3A). FSTL3 expression was lowest in control GES1 cell line; however, FSTL3 levels were raised in all gastric cancer cell lines with the highest expression in MGC-803 and lowest in SGC-7901 cell lines (Figure 3A). Further analysis of the endogenous FSTL3 mRNA levels using qRT-PCR further supported this trend, with SGC-7901 showing 1.5-fold rise compared to 4-fold rise in MGC-803 cells (Figure 3B). These 2 lines were then selected as low and high FSTL3 expressors for testing FSTL3 regulation of gastric cancer cell function. In order to explore whether FSTL3 regulates gastric cancer cell function, we overexpressed FSTL3 in SGC-7901 cells (Figure 3C). We showed that this FSTL3 plasmid caused 3-fold rise in FSTL3 mRNA levels; furthermore, this correlated with an increase in FSTL3 protein levels (Figure 3C). We then made a lentiviral system to express small hairpin RNAs (shRNAs) that target the FSTL3 mRNA; screening 3 different FSTL3-specific shRNAs showed that sh-1 was most effective (Figure 3D). FSTL3 mRNA levels were reduced by $60 \%$ using sh-1; a similar knockdown in FSTL3 protein levels was also evident (Figure 3D).

We then assessed FSLT3 overexpression or knockdown on gastric cancer cell responses (Figure 3E-3H). Measurement of cell viability showed increased FSTL3 levels promoted gastric cancer cell line SGC-7901 viability; in contrast, FSTL3 knockdown caused decreased cell viability in MGC-803 (Figure 3E). Measurement of new DNA synthesis using EdU incorporation showed 25\% increased cell proliferation upon FSTL3 overexpression in gastric cancer cell line SGC-7901 (Figure 3F). Again, FSTL3 knockdown caused 25\% decrease in MGC-803 cell proliferation (Figure 3F). We then asked whether FSTL3 overexpression or knockdown affected cell monolayer closure, which is dependent on both cell proliferation and migration (Figure 3G). FSTL3 overexpression promoted a $20 \%$ increase in monolayer wound closure compared to control in gastric cancer cell line SGC-7901 (Figure 3G). However, FSTL3 knockdown promoted a $30 \%$ decrease in monolayer wound closure compared to control in gastric cancer cell line MGC-803 (Figure 3G).

We also assessed FSTL3 overexpression or knockdown on cell migration using the Transwell assay (Figure $3 \mathrm{H})$. FSTL3 overexpression promoted a 2 -fold increase in cell migration compared to control in gastric cancer cell line SGC-7901 (Figure 3H). However, FSTL3 knockdown promoted a $60 \%$ decrease in cell migration compared to control in gastric cancer cell line MGC803 (Figure $3 \mathrm{H}$ ). We also assessed FSTL3 to the formation of cell colonies in culture, an important feature of tumor growth (Figure 3I). FSTL3 knockdown caused 50\% decrease in gastric cancer cell line MGC803 colony formation compared to control (Figure 3I). FSTL3 was also overexpressed in the normal gastric epithelial cell line, GES1 (Supplementary Figure 1A). This showed 2-fold increase in FSTL3 mRNA (Supplementary Figure 1B) and protein (Supplementary Figure 1A) levels. Increased FSTL3 expression promoted increased GES1 cell viability (Supplementary Figure 1C). Analysis of EdU incorporation showed 30\% increase in new DNA synthesis in FSTL3overexpressing cells compared to control (Supplementary Figure 1D). Analysis of GES1 FSTL3overexpressing cells revealed $75 \%$ increase in colony formation compared to control (Supplementary Figure 1E). To further test inference from our bioinformatics studies, we knocked down and overexpressed the expression of FSTL3 in gastric cancer cells. It was found that the over-expression of FSTL3 promotes cell 
adhesion and the number of focal adhesion spots (Supplementary Figure 2A, 2B).

\section{FSTL3 promotes gastric cancer cell tumorigenicity in vivo}

We then assessed the contribution of FSTL3 to gastric cancer cell tumorigenicity using an in vivo animal model
(Figure 4). The MGC-803 gastric cancer cell line expressing the sh-FSTL3 construct with stable FSTL3 knockdown was compared to the parental cell line (control) for the ability to promote tumor development in the immunocompromised Nude mouse model. Bioimaging of MC-803-derived tumors in Nude mice showed that tumor size was reduced (Figure 4A). Tumors were excised and examined visually: again, FSTL3
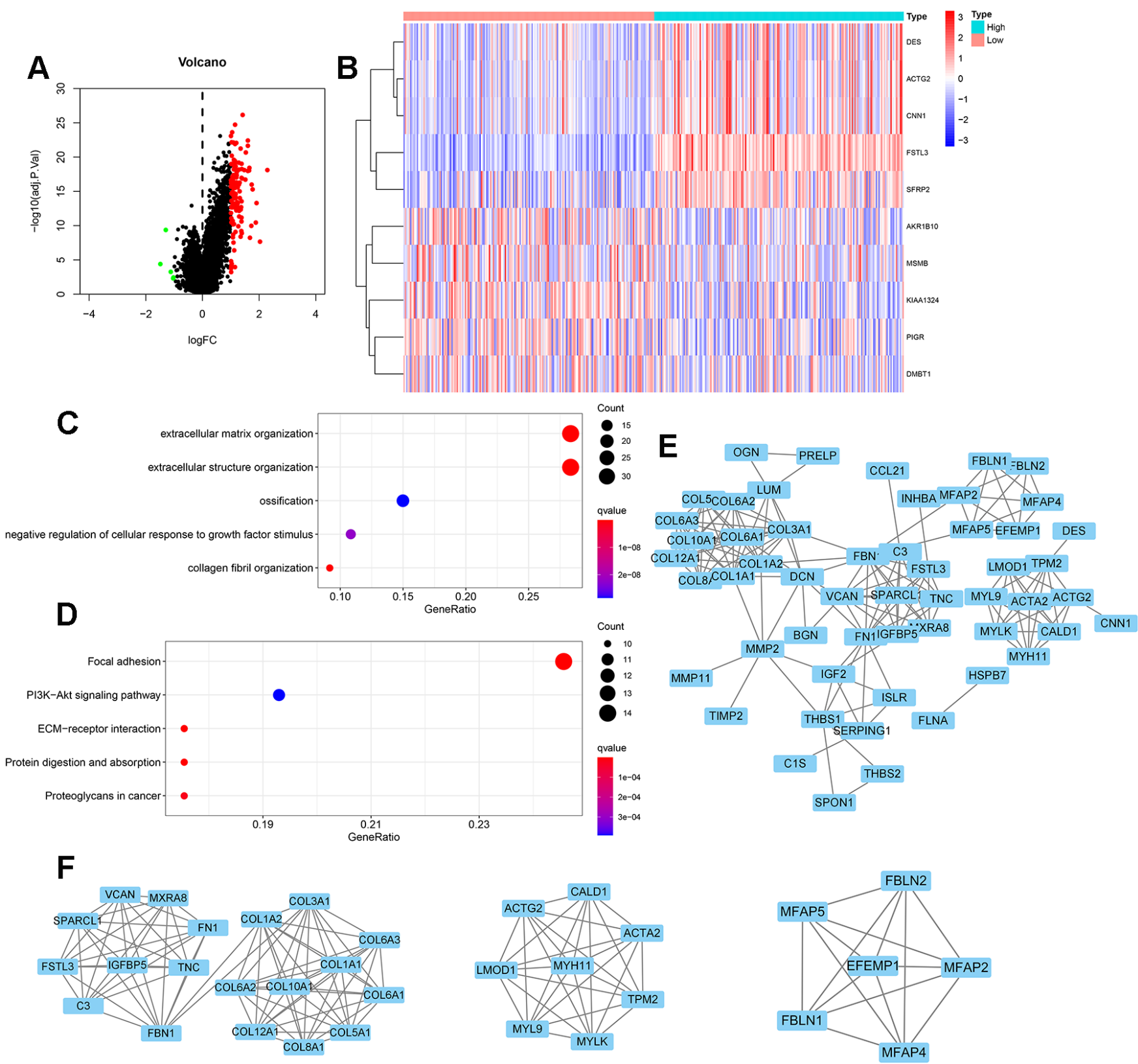

Figure 2. Differential gene expression and functional enrichment during FSTL3 overexpression. (A) Volcano map of differentially expressed genes under conditions of elevated FSTL3 levels; adjusted $P<0.05$. (B) The top 10 differentially expressed genes (linked to FSTL3 overexpression) in gastric cancer disease presented as a gene expression heat map. (C) Gene ontology (GO) term enrichment analysis; adjusted $P<0.05$. (D) Kyoto Encyclopedia of Genes and Genomes (KEGG) biochemical pathway enrichment analysis; adjusted $P<0.05$. (E) Protein-protein interaction (PPI) network analysis for 122 differentially expressed genes linked to gastric cancer; minimum required interaction score: 0.900. (F) Specific PPI nodes within the full network with MYOCD tool in Cytoscape. 
knockdown suggested smaller tumor size compared to parental MGC-803-derived tumors (Figure 4B). Further analysis revealed $50 \%$ reduction in tumor weight upon FSTL3 knockdown (Figure 4C). There was 30\% reduction in tumor volume upon FSTL3 knockdown (Figure 4C). Hematoxylin-eosin staining of tumor sections suggested a more irregular cell shape and packing in tumors from the parent cell line MGC-803derived tumor compared to FSTL3 knockdown conditions (Figure 4D). Analysis of the cell proliferation marker, Ki67, showed a much higher immunoreactivity for nuclear Ki67 in parent cell line MGC-803-derived tumors compared to FSTL3 knockdown tumors (Figure 4D). We then probed for
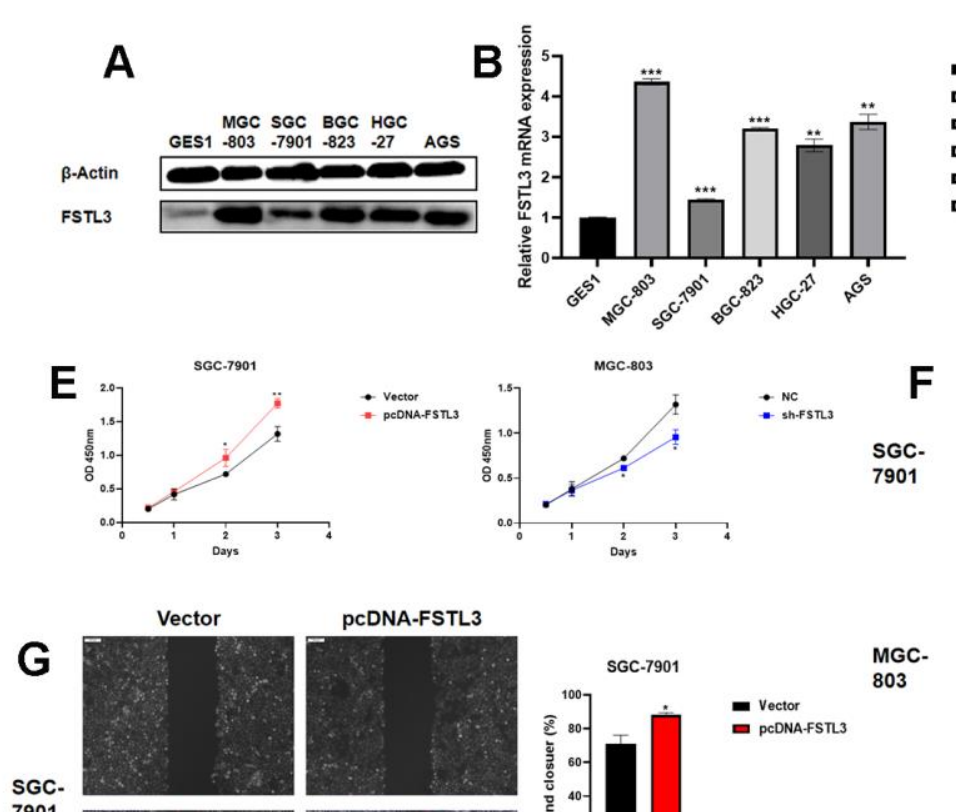

7901
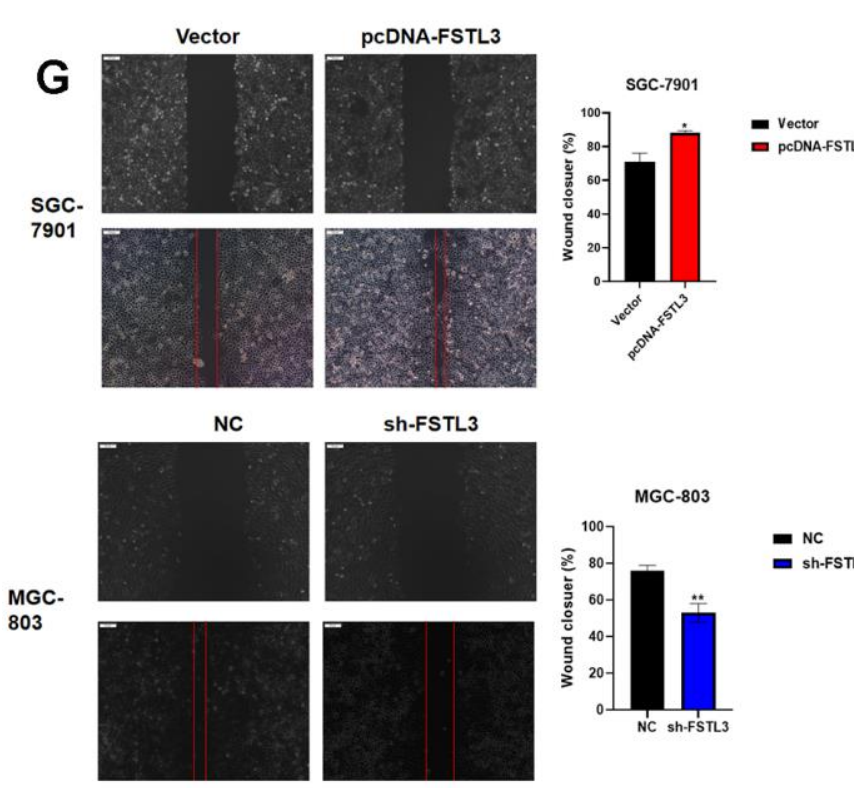
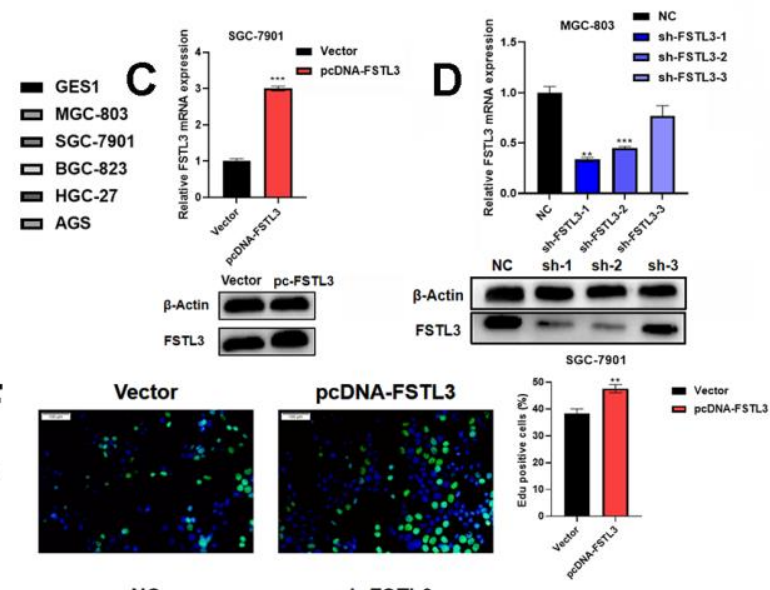

NC
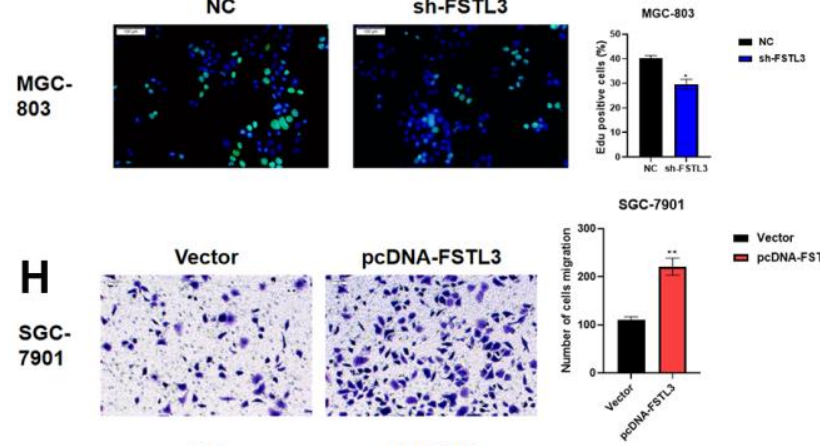

- Vector
pCDNA.FSTLS
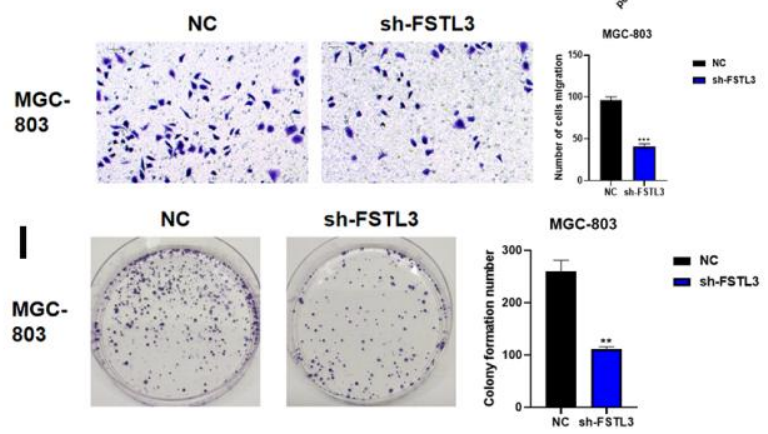

- NC

= ${ }_{\text {sh-FSTL3 }}^{\text {NC }}$

Figure 3. FSTL3 overexpression or knockdown modulates gastric cancer cell viability, proliferation and migration. (A) Western blot analysis of FSTL3 protein levels in GES1, MGC-803, SGC-7901, BGC-823, HGC-27 and AGS gastric cell lines; blotting for the housekeeping protein $\beta$-actin was used as a loading control in this experiment. (B) Analysis of relative FSTL3 mRNA levels in GES1, MGC-803, SGC-7901, BGC-823, HGC-27 and AGS gastric cell lines using qRT-PCR (see Materials and Methods). (C) Overexpression of FSTL3 in gastric cancer cell line SGC-7901 monitored by Western blotting and qRT-PCR. (D) FSTL3 knockdown in gastric cancer cell line MGC-803 monitored by Western blotting and qRT-PCR. (E) FSTL3 overexpression (SGC-7901) or knockdown (MGC-803) cell lines were analyzed for cell viability compared to controls. (F) FSTL3 overexpression (SGC-7901) or knockdown (MGC-803) cell lines were assayed for cell proliferation using EdU incorporation. (G) Wounded cell monolayer assays on FSTL3 overexpression (SGC-7901) or knockdown (MGC-803) cell lines (48h). (H) Transwell cell migration assay on FSTL3 overexpression (SGC-7901) or knockdown (MGC-803) cell lines followed by staining of Transwell filters to detect migrated cells. (I) FSTL3 knockdown and effects on MGC-803 cell colony formation. Quantification in panels (B-I) were carried out as described in Materials and Methods and compared to control cell lines. Error bars indicate \pm SEM; significance indicated by asterisks, ${ }^{*} P<0.05$, $* * P<0.01, * * * P<0.001$; number of experiments, $\mathrm{n}=3$. 
FSTL3 expression in these tumors using Western blotting, and found 70\% decrease in FSTL3 mRNA levels correlated with decreased FSTL3 protein levels in MGC-803 knockdown cells (Figure 4E). Under these condition, stable FSTL3 knockdown caused 30\% decrease in expression of cell proliferation marker, Ki67 (Figure 4E).

We then assessed the effects on tumor metastasis in vivo using tail vein administration of MGC-803 parental line or MGC-803/sh-1 cell into Nude mice. After allowing tumors to develop and spread systemically, we analyzed the frequency of mouse lung tissues for secondary tumor metastases. There were both 2 deaths in the control group and FSTL3 knockdown group of nude mice. Bioluminescence imaging results revealed that FSTL3 knockdown significantly reduced incidence in the lung of metastatic secondary tumors (Figure 4F, 4G). The expression levels of FSTL3 thus influences both cell proliferation and tumor progression.

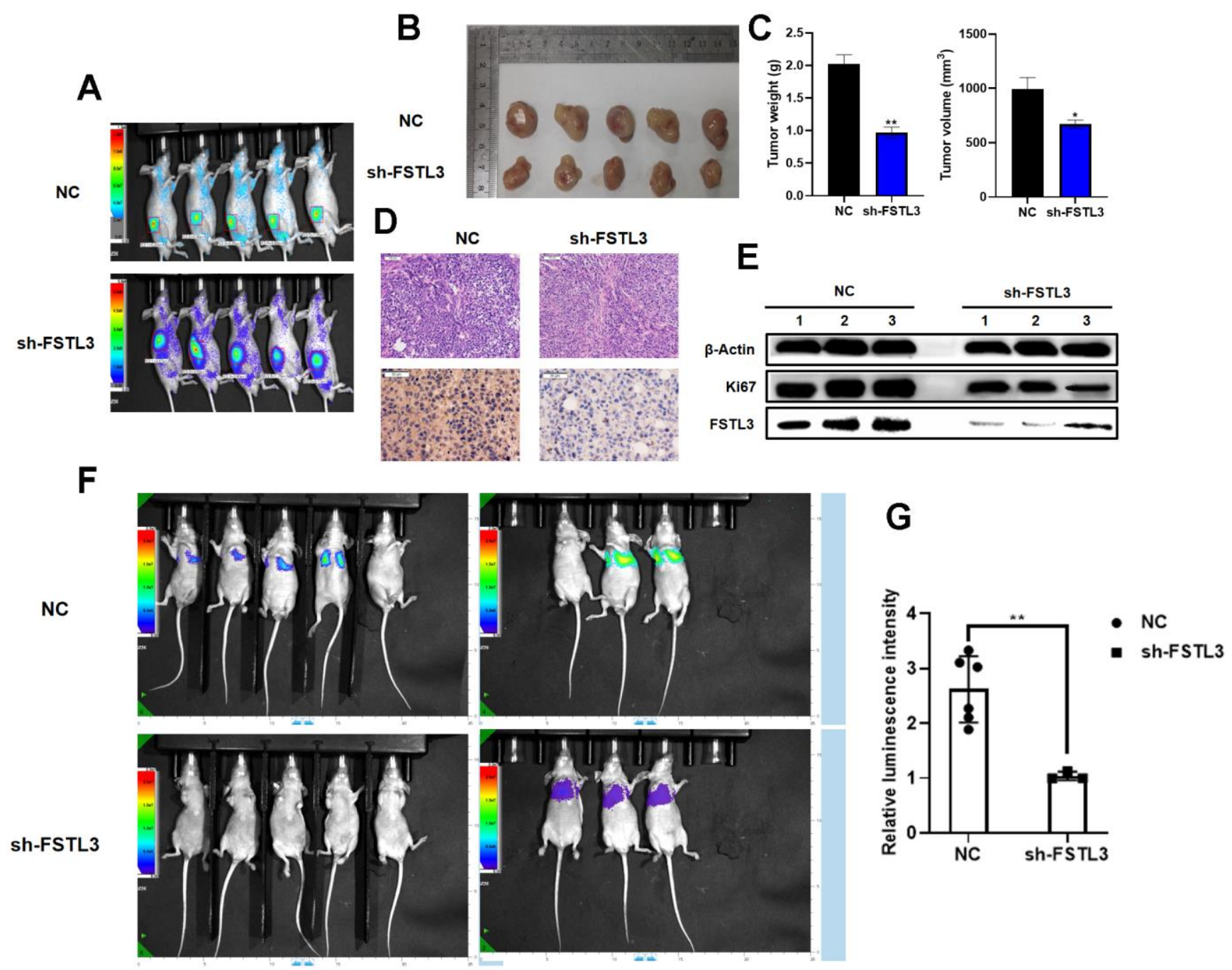

Figure 4. FSTL3 expression promotes gastric cancer tumorigenicity in vivo. (A) Bioimaging of MGC-803-derived tumors in the flanks of Nude mice. Growth of tumors in Nude mice injected for control MGC-803 cells (NC) or cells expressing shRNA-specific for FSTL3 (sh-FSTL3). (B) Excision and viewing of subcutaneous tumors derived from Nude mice injected for control MGC-803 cells (NC) or cells expressing shRNAspecific for FSTL3 (sh-FSTL3). (C) Analysis of weight or volume of tumors derived from Nude mice injected for control MGC-803 cells (NC) or cells expressing shRNA-specific for FSTL3 (sh-FSTL3). (D) Hematoxylin-eosin (upper panels) or Ki67 immunohistochemistry (lower panels) on tissue sections from subcutaneous tumors derived from Nude mice injected for control MGC-803 cells (NC) or cells expressing shRNA-specific for FSTL3 (sh-FSTL3). (E) Analysis of FSTL3 and Ki67 using Western blotting of tumors from Nude mice injected for control MGC-803 cells (NC) or cells expressing shRNA-specific for FSTL3 (sh-FSTL3). Blotting of tumor lysates using antibodies to FSTL3 or Ki67; antibodies to $\beta$-actin were used to check for protein loading in tumor lysates. (F) Bioluminescence imaging results of the lung metastasis frequency from Nude mice injected for control MGC-803 cells (NC) or cells expressing shRNA-specific for FSTL3 (sh-FSTL3). (G) Statistical analysis of luminescence intensity. Error bars indicate $+\mathrm{SEM}$; significance indicated by asterisks, ${ }^{*} P<0.05,{ }^{* *} P<0.01, * * * P<0.001$; number of experiments, $\mathrm{n}=3$. 


\section{FSTL3 mRNA is a target of miR-486-5p}

To evaluate whether mRNA turnover was a potential regulatory mechanism to control FSTL3 protein levels, we used StarBase (https://web.archive.org/web/ 20110222111721/http://starbase.sysu.edu.cn/) to look for potential miRNAs that bind to FSTL3. Using this approach, we identified an miRNA termed miR-486-5p as a potential regulator which binds to a site in the 3'UTR of the FSTL3 mRNA (Figure 5A). To assess the correlation between miR-486-5p, FSTL3 and cancer status, we used the STAD database within TCGA. We found that miR486-5p levels were significantly decreased in gastric cancer patients compared to controls (Figure 5B). Further analysis showed a correlation between decreased miR486-5p levels with increased FSTL3 levels (Figure 5C).
We then examined miR-486-5p levels in normal gastric epithelial cells (GES1) vs. gastric cancer cell lines, MGC803 and SGC-7901, using qRT-PCR (Figure 5D). There was a significant 20-30\% decrease in miR-486-5p levels in gastric cancer cell lines (MGC-803, SGC-7901) compared to normal gastric cells (GES1).

To further verify whether FSTL3 is indeed a target of miR-486-5p we constructed a synthetic miR-486-5p mimic. Transfection of this miR-486-5p mimic was transfected into gastric cancer cell line MGC-803: FSTL3 expression was decreased 50\% (Figure 5E). We also made a synthetic miR-486-5p inhibitor, and delivery of this into gastric cancer cells caused a 1.5-2-fold rise in FSTL3 protein levels; this correlated with a 2.5 -fold rise in FSTL3 mRNA levels (Figure 5E). We

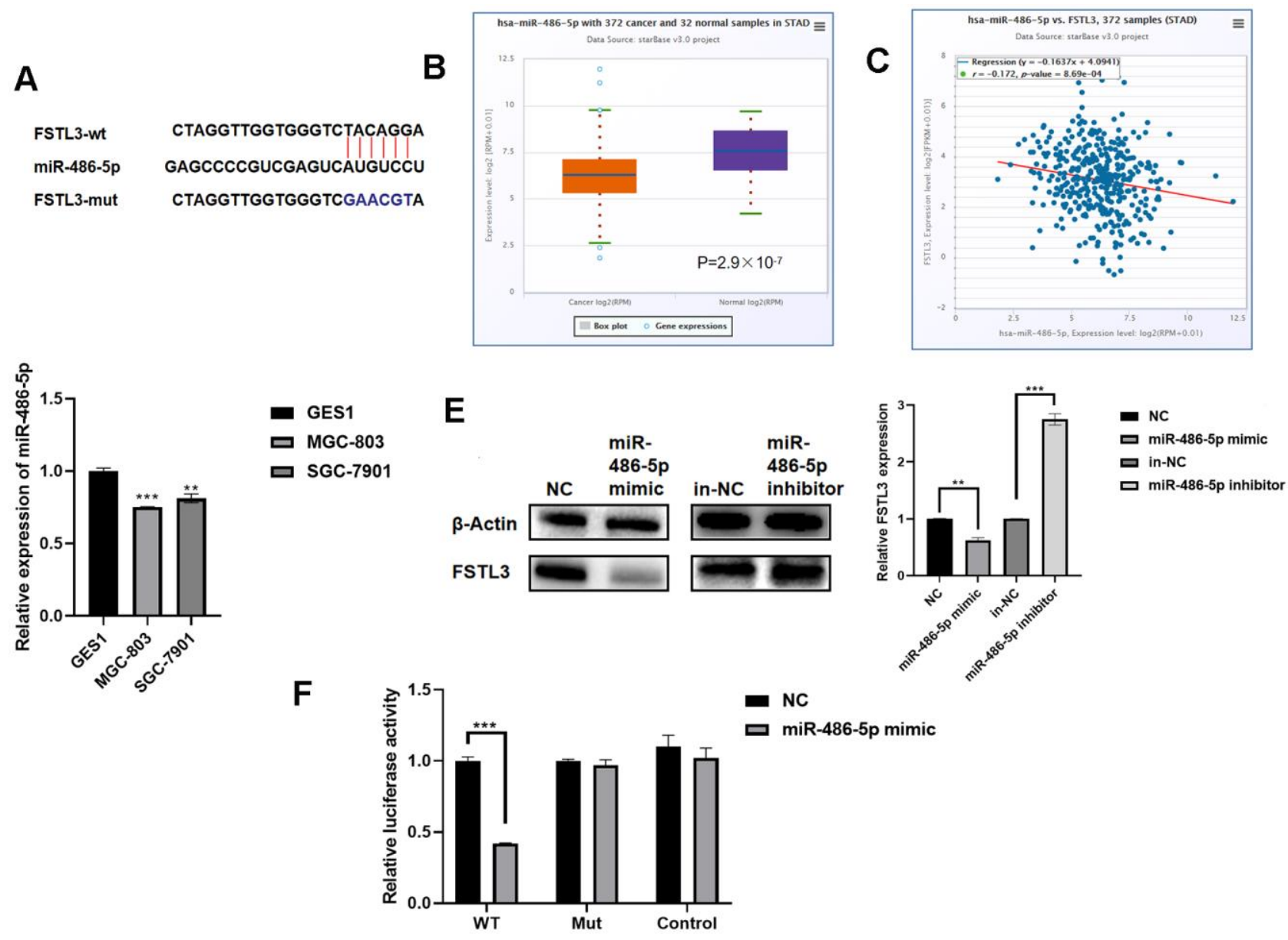

Figure 5. FSTL3 mRNA is regulated by miR-486-5p levels. (A) Schematic depiction of the predicted binding site for miR-486-5p in the FSTL3 mRNA. (B) Analysis of miR-486-5p levels in gastric cancer tumors vs. normal tissues in the STAD database in TCGA. (C) Correlative analysis of miR-486-5p and FSTL3 levels in gastric cancer tumors using the STAD database. (D) Profiling of miR-486-5p levels in normal and cancer gastric cancer cell lines using qRT-PCR. (E) Western blotting was used to analyze protein and RNA levels in gastric cancer MGC-803 cells subjected to transfection with miR-486-5p mimic or miR-486-5p inhibitor. (F) A dual luciferase assay was carried out using transfection of luciferase plasmid constructs alongside miR-486-5p mimic or miR-486-5p inhibitor. HEK 293T cells co-transfected with Luc-FSTL3-wt (WT) or Luc-FSTL3-mut (MUT) plasmids with synthetic miR-486-5p constructs. Error bars indicate \pm SEM; significance indicated by asterisks, ${ }^{*} P<0.05, * * P<0.01, * * * P<0.001$; number of experiments, $\mathrm{n}=3$. 
also used a dual luciferase reporter assay where the luciferase cDNA was fused to either the wild-type FSLT3 3'UTR (FSLT3-wt) or a mutant FSLT3 3'UTR that was defective for binding miR-486-5p. Cotransfection of miR-486-5p mimic and luciferase FSTL3-wt showed 60\% reduction in luciferase activity (Figure 5F). In contrast, co-transfection of miR-486-5p mimic and luciferase FSTL3-mut showed no effect on luciferase activity (Figure 5F). These data suggest that miR-486-5p directly regulates FSTL3 mRNA stability and/or degradation.

\section{MiR-486-5p specificity for FSTL3 mRNA and effects on gastric cancer cell responses}

To understand the link between FSTL3 mRNA and miR-486-5p, we evaluated whether such potential regulation impacts on gastric cancer cell responses. We focused on the miR-486-5p inhibitor and its ability to modulate FSTL3 knockdown by shRNA (Figure 6). We transfected alone or co-transfected the shRNA specific for FSTL3 and/or miR-486-5p inhibitor in the MGC803 cell line followed by Western blotting (Figure 6A). The FSTL3 protein levels were knocked down by shFSTL3, however, this effect could be partially reversed by miR-486-5p inhibitor (Figure 6A, 6B). The miR486-5p inhibitor caused a substantial 2-fold rise in FSTL3 mRNA levels (Figure 6B). The sh-FSTL3 construct caused $60 \%$ knockdown of FSTL3 mRNA levels; however, this was partially reversed by miR486-5p inhibitor with 2-fold rise in FSTL3 mRNA levels (Figure 6B).

We then assessed the effects of targeting miR-486-5p on MGC-803 gastric cancer cell responses. The miR486-5p inhibitor caused a substantial rise in new proliferating cells compared to control (NC) cells, shown by EdU staining (Figure 6C). The sh-FSTL3 construct caused reduced nuclear EdU staining; however, this was reversed by miR-486-5p inhibitor (Figure 6C). Using the wounded cell monolayer closure assay, we demonstrated that control (NC) cells show wounded monolayer closure after $24 \mathrm{~h}$ (Figure 6D). Transfection of miR-486-5p inhibitor caused a dramatic increase in wounded monolayer closure; this miR-486$5 \mathrm{p}$ inhibitor caused a partial reversal in the inhibitory effects mediated by sh-FSTL3 construct in this assay (Figure 6D). Similar conclusions were reached in the analysis of cell migration using the Transwell assay (Figure 6E). Transfection of miR-486-5p inhibitor caused a dramatic increase in MGC-803 cell migration; furthermore, miR-486-5p inhibitor and sh-FSTL3 treatment together caused a partial reversal of the inhibitory effects on MGC-803 cell migration caused by sh-FSTL3 construct treatment alone (Figure 6D). Such findings further support the function role of miR-486-5p inhibitor in controlling FSTL3 levels and gastric cell responses.

\section{DISCUSSION}

The follistatin (FST) family of secreted glycoproteins are increasingly associated with different types of cancers. In a functional analysis of a family member, termed FSTL3, we provide evidence that a mechanism which directly modulates FSTL3 mRNA levels directly impacts on gastric cancer cell responses which contribute to cancer development and progression. This is based on 4 lines of evidence. First, cancer bioinformatics shows a clear association with increased FSTL3 expression in gastric cancer patients. Furthermore, high FSTL3-expressing gastric cancer patients have reduced survival over a $10 \mathrm{yr}$ period. Second, modulation of FSTL3 expression in a humanmouse tumor xenograft model, suggests that higher levels of FSTL3 are linked to increased tumor growth and spread. Third, modulation of FSTL3 shows a clear link with gastric cell responses such as cell viability, proliferation and migration. Finally, FSTL3 mRNA is regulation by miR-486-5p modulates FSTL3 expression and gastric cancer cell-associated responses.

FSTL3 is a secreted glycoprotein encoded by a gene located on human chromosome 19q13.3. FSTL3 was originally discovered as a rearranged gene locus associated with some blood disorders and malignancies [12]. The follistatin (FST) family members are single-chain monomeric glycoproteins which potentially interact with other soluble factors and/or membrane receptors [27]. Follistatin can bind to members of the TGF- $\beta$ superfamily (e.g. activin); it can also bind FSH and inhibits its biological activity. Another family member, FSTL1 is associated with reduced levels in prostate cancer; FSTL1 expression correlates with inflammatory factors and transforming factors [28]. In gastric cancer, it was reported that FSTL1 knockdown promotes apoptosis via the STAT6 signaling pathway [29]. In hepatocellular carcinoma, FSTL5 expression inhibits the HCC growth in vitro by inducing apoptosis [30].

miRNA is a single-stranded non-coding RNA (18-25 nucleotides) endogenously encoded which regulates protein translation or mRNA degradation via interaction with the 3'or 5' untranslated regions (UTRs) of target mRNAs [31]. Many cell biological processes such as cell proliferation, growth, differentiation, apoptosis and the cell cycle are regulated by a variety of miRNAs [32]. miR-486-5p is a recently discovered miRNA which displays abnormal expression in colon cancer [33], gastric cancer [34], liver cancer [35] and other malignant tumors; miR-486-5p levels are linked to 
A

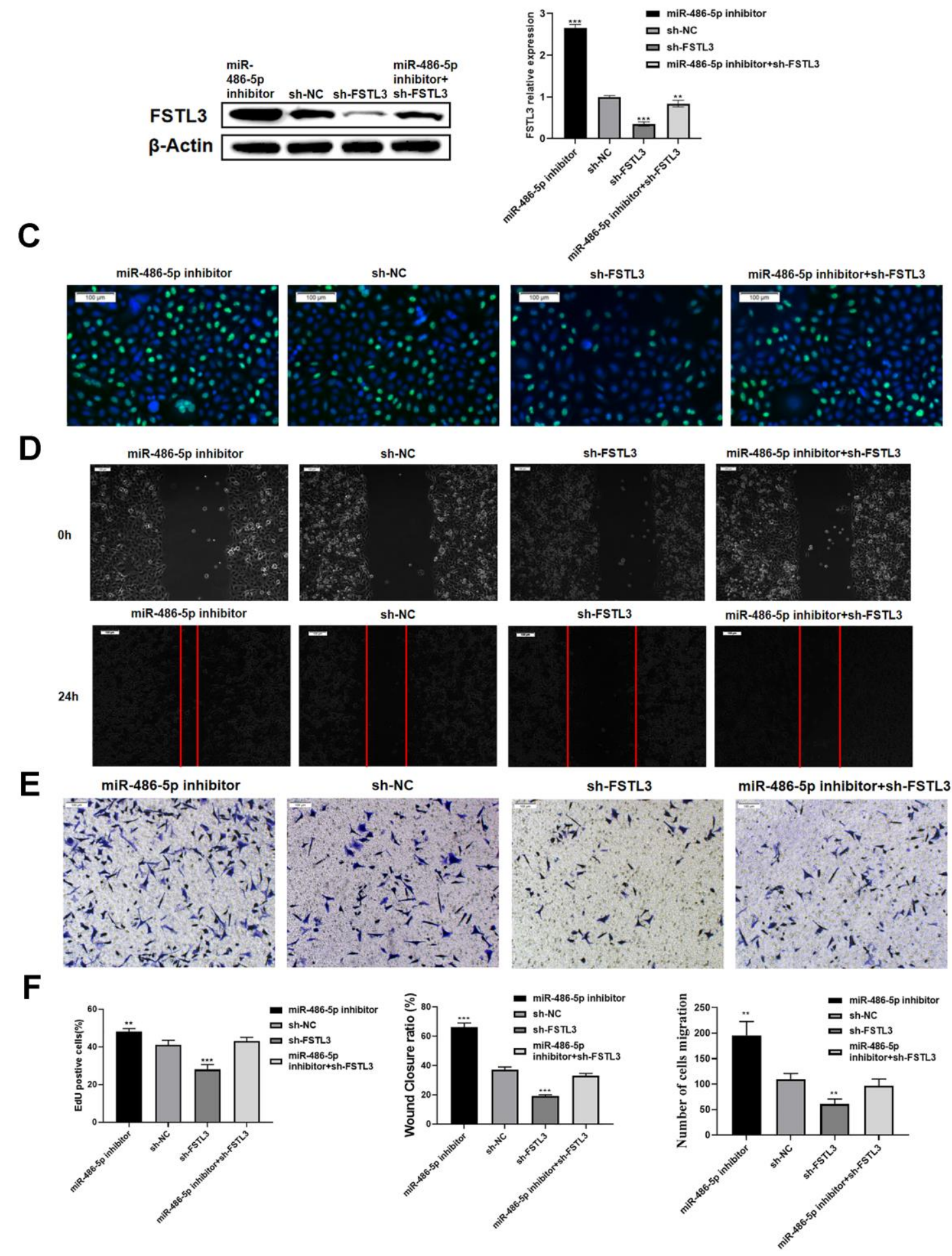

Figure 6. Evaluating miR-486-5p specificity for FSTL3 mRNA. (A) Western blotting evaluation of FSTL3 protein levels after transfection of sh-FSTL3 and/or miR-486-5p inhibitor into MGC-803 gastric cancer cells. Monitoring $\beta$-actin levels was used as an internal control in these blots. (B) Western blot results were quantified by using Image J software. Error bars indicate \pm SEM; significance indicated by asterisks, ${ }^{* *} P<0.01, * * * P<0.001$; number of experiments, $\mathrm{n}=3$. (C) Transfection of sh-FSTL3 and/or miR-486-5p inhibitor into MGC-803 gastric cancer cells and evaluation of cell proliferation using EdU assay (24h). (D) Wounded cell monolayer closure assay after transfection of sh-FSTL3 and/or miR-486-5p inhibitor followed by imaging at 8 and $24 \mathrm{~h}$ post-wounding. (E) The Transwell assay for cell migration after transfection of sh-FSTL3 and/or miR-486-5p inhibitor was into MGC-803 cells. Sh-NC construct (shRNA) is a negative control for FSTL3 knockdown. (F) Quantified results of EdU, wound healing and transwell migration assays. Sh-NC was analyzed as a control. 
tumor growth and invasion. In gastric adenocarcinoma, miR-486-5p is a potential tumor suppressor. Comparison of normal gastric epithelial cells and tissues vs. gastric cancer cell lines and tumors, and is miR-486-5p is a combinatorial risk factor alongside olfactomedin-4 (OLFM4) and fibroblast growth factor 9 (FGF9). The combination of FGF9 expression alongside other genes can inhibit gastric cancer cells proliferation and is an independent risk factor for predicting gastric cancer patient survival $[34,36]$. In this study, we found that by combining in silico bioinformatics with cellular experiments, miR-486-5p levels were down-regulated in gastric cancer cell lines and tumors. This is supported by previous studies elsewhere [34]. We further delineated the potential site of interaction between miR486-5p with the 3'UTR of the FSTL3 mRNA. Overexpression of a synthetic miR-486-5p mimic causes FSTL3 down-regulation; however, use of a synthetic miR-486-5p inhibitor causes a rise in FSTL3 levels. Finally, miR-486-5p and FSTL3 levels correlate negatively in an analysis of gene expression in gastric cancer tissues.

What is the functional target of FSTL3? One possibility is that FSTL3 binds to a cell surface receptor which stimulates autocrine signaling and cellular responses linked to cell proliferation and migration. Alternatively, as noted for the FST founding family member capacity to bind other secreted factors, FSTL3 may sequester autoinhibitory factors thus effecting a net autocrine stimulatory effect. Our findings provide a new framework to better understand gastric cancer development and progression and develop new therapeutic strategies. However, there are also specific limitations of our study. First, the incidence of Asian and African patients within the TCGA database is low, with skewed view of the molecular events underlying cancer processes. Whether FSTL3 is a new diagnostic biomarker of gastric cancer requires the collection of more unbiased clinical datasets across the global population. Next, FSTL3 mRNA may be the target of multiple miRNAs, and more work is needed to assess such factors. The role of miR-486-5p as a regulator of FSTL3 miRNA degradation needs to be checked for its ability to target other cellular mRNA species. Our work clearly supports a role for FSTL3 in promoting gastric cancer cell proliferation and migration; furthermore, FSTL3 expression contributes to gastric tumor growth, invasion and metastasis. FSTL3 shows promise as a new biomarker for gastric cancer diagnosis and is a new therapeutic target in this disease.

\section{Abbreviations}

miRNA: microRNA; 3'-UTR: 3'untranslated region; TCGA: The Cancer Genome Atlas; GEO: Gene
Expression Omnibus; PPI: Protein-protein interaction; STAD: stomach adenocarcinoma.

\section{AUTHOR CONTRIBUTIONS}

X.H.L. designed research; X.H.L., Y.X., S.C. Y.H. and Z.T.D. performed research., J.P.L., H.M.Z., Z.T.D., and Q.B.Z. analyzed data; and Q.F.W, Z.T.D, S.P. wrote and revised the manuscript. All authors read and approved the final draft of the manuscript.

\section{CONFLICTS OF INTEREST}

The authors declare that they have no conflicts of interest.

\section{FUNDING}

This work was supported by the National Natural Science Foundation of China (No. 31501149, 31770815, 31570764), Hubei Natural Science Foundation (2017CFB537), Educational Commission of Hubei (B2020001) and Royal Society International Exchanges UK-China Award IECINSFCl181262.

\section{REFERENCES}

1. Siegel RL, Miller KD, Jemal A. Cancer statistics, 2020. CA Cancer J Clin. 2020; 70:7-30. https://doi.org/10.3322/caac.21590 PMID:31912902

2. Jiang Y, Ajani JA. Multidisciplinary management of gastric cancer. Curr Opin Gastroenterol. 2010; 26:640-46. https://doi.org/10.1097/MOG.0b013e32833efd9b PMID:20827183

3. Leung $\mathrm{WK}, \mathrm{Ho} \mathrm{HJ}$, Lin JT, Wu MS, Wu CY. Prior gastroscopy and mortality in patients with gastric cancer: a matched retrospective cohort study. Gastrointest Endosc. 2018; 87:119-27.e3. https://doi.org/10.1016/j.gie.2017.06.013 PMID:28648576

4. Zhang $X$, Li M, Chen S, Hu J, Guo Q, Liu R, Zheng H, Jin Z, Yuan Y, Xi Y, Hua B. Endoscopic Screening in Asian Countries Is Associated With Reduced Gastric Cancer Mortality: A Meta-analysis and Systematic Review. Gastroenterology. 2018; 155:347-54.e9. https://doi.org/10.1053/j.gastro.2018.04.026 PMID:29723507

5. Digklia A, Wagner AD. Advanced gastric cancer: Current treatment landscape and future perspectives. World J Gastroenterol. 2016; 22:2403-14. https://doi.org/10.3748/wjg.v22.i8.2403 PMID:26937129 
6. Wang $Q$, Zhang $X$, Shen E, Gao J, Cao F, Wang X, Li Y, Tian T, Wang J, Chen Z, Wang J, Shen L. The anti-HER3 antibody in combination with trastuzumab exerts synergistic antitumor activity in HER2-positive gastric cancer. Cancer Lett. 2016; 380:20-30.

https://doi.org/10.1016/i.canlet.2016.06.005 PMID:27317872

7. Wagner AD, Syn NL, Moehler M, Grothe W, Yong WP, Tai BC, Ho J, Unverzagt S. Chemotherapy for advanced gastric cancer. Cochrane Database Syst Rev. 2017; 8:CD004064.

https://doi.org/10.1002/14651858.CD004064.pub4 PMID:28850174

8. Gravalos C, Jimeno A. HER2 in gastric cancer: a new prognostic factor and a novel therapeutic target. Ann Oncol. 2008; 19:1523-29.

https://doi.org/10.1093/annonc/mdn169

PMID:18441328

9. Deng JY, Liang H. Clinical significance of lymph node metastasis in gastric cancer. World J Gastroenterol. 2014; 20:3967-75.

https://doi.org/10.3748/wjg.v20.i14.3967

PMID:24744586

10. Pan JH, Zhou H, Cooper L, Huang JL, Zhu SB, Zhao XX, Ding $H$, Pan YL, Rong L. LAYN Is a Prognostic Biomarker and Correlated With Immune Infiltrates in Gastric and Colon Cancers. Front Immunol. 2019; 10:6.

https://doi.org/10.3389/fimmu.2019.00006 PMID:30761122

11. Kim J, Bae DH, Kim JH, Song KS, Kim YS, Kim SY. HOXC10 overexpression promotes cell proliferation and migration in gastric cancer. Oncol Rep. 2019; 42:202-12.

https://doi.org/10.3892/or.2019.7164 PMID:31115563

12. Hayette S, Gadoux M, Martel S, Bertrand S, Tigaud I, Magaud JP, Rimokh R. FLRG (follistatin-related gene), a new target of chromosomal rearrangement in malignant blood disorders. Oncogene. 1998; 16:2949-54.

https://doi.org/10.1038/sj.onc.1201807 PMID: 9671416

13. Namdari M, Negahdari B, Cheraghi M, Aiyelabegan HT, Eatmadi A. Cardiac failure detection in 30 minutes: new approach based on gold nanoparticles. J Microencapsul. 2017; 34:132-39.

https://doi.org/10.1080/02652048.2017.1296900 PMID:28264603

14. Kelaini $S$, Vilà-González $M$, Caines $R$, Campbell $D$, Eleftheriadou M, Tsifaki M, Magee C, Cochrane A, O'neill K, Yang C, Stitt AW, Zeng L, Grieve DJ, Margariti A. Follistatin-Like 3 Enhances the Function of Endothelial Cells Derived from Pluripotent Stem Cells by Facilitating $\beta$-Catenin Nuclear Translocation Through Inhibition of Glycogen Synthase Kinase-3 $\beta$ Activity. Stem Cells. 2018; 36:1033-44. https://doi.org/10.1002/stem.2820 PMID:29569797

15. Barrios-Silva LV, Parnell M, Shinwari ZB, Chaudhary GA, Xenofontos T, van Bekhoven A, McArthur S, Elliott BT. Activin subfamily peptides predict chronological age in humans. Physiol Rep. 2018; 6:e13823.

https://doi.org/10.14814/phy2.13823 PMID:30178598

16. Runhua M, Qiang J, Yunqing S, Wenjun D, Chunsheng W. FSTL3 Induces Lipid Accumulation and Inflammatory Response in Macrophages and Associates With Atherosclerosis. J Cardiovasc Pharmacol. 2019; 74:566-73.

https://doi.org/10.1097/FJC.0000000000000742 PMID:31815869

17. Panagiotou G, Papakonstantinou E, Vagionas A, Polyzos SA, Mantzoros CS. Serum Levels of Activins, Follistatins, and Growth Factors in Neoplasms of the Breast: A Case-Control Study. J Clin Endocrinol Metab. 2019; 104:349-58.

https://doi.org/10.1210/jc.2018-01581

PMID:30388235

18. Razanajaona D, Joguet S, Ay AS, Treilleux I, GoddardLéon S, Bartholin L, Rimokh R. Silencing of FLRG, an antagonist of activin, inhibits human breast tumor cell growth. Cancer Res. 2007; 67:7223-29.

https://doi.org/10.1158/0008-5472.CAN-07-0805 PMID: 17671190

19. Herbst RS, Ansari R, Bustin F, Flynn P, Hart L, Otterson GA, Vlahovic G, Soh CH, O'Connor P, Hainsworth J. Efficacy of bevacizumab plus erlotinib versus erlotinib alone in advanced non-small-cell lung cancer after failure of standard first-line chemotherapy (BeTa): a double-blind, placebo-controlled, phase 3 trial. Lancet. 2011; 377:1846-54.

https://doi.org/10.1016/S0140-6736(11)60545-X PMID:21621716

20. Esteller M. Non-coding RNAs in human disease. Nat Rev Genet. 2011; 12:861-74. https://doi.org/10.1038/nrg3074 PMID:22094949

21. Hao NB, He YF, Li XQ, Wang K, Wang RL. The role of miRNA and IncRNA in gastric cancer. Oncotarget. 2017; 8:81572-82.

https://doi.org/10.18632/oncotarget.19197 PMID:29113415

22. Zheng $P$, Chen L, Yuan X, Luo Q, Liu Y, Xie G, Ma Y, Shen L. Exosomal transfer of tumor-associated macrophagederived miR-21 confers cisplatin resistance in gastric cancer cells. J Exp Clin Cancer Res. 2017; 36:53. https://doi.org/10.1186/s13046-017-0528-y PMID:28407783 
23. Adami B, Tabatabaeian H, Ghaedi K, Talebi A, Azadeh $M$, Dehdashtian E. miR-146a is deregulated in gastric cancer. J Cancer Res Ther. 2019; 15:108-14.

https://doi.org/10.4103/icrt.JCRT 85517 PMID:30880764

24. Cancer Genome Atlas Research Network. Comprehensive genomic characterization defines human glioblastoma genes and core pathways. Nature. 2008; 455:1061-68.

https://doi.org/10.1038/nature07385 PMID: 18772890

25. Li JH, Liu S, Zhou $H$, Qu LH, Yang JH. starBase v2.0: decoding miRNA-ceRNA, miRNA-ncRNA and proteinRNA interaction networks from large-scale CLIP-Seq data. Nucleic Acids Res. 2014; 42:D92-97.

https://doi.org/10.1093/nar/gkt1248

PMID:24297251

26. Szklarczyk $D$, Franceschini $A$, Kuhn $M$, Simonovic $M$, Roth A, Minguez P, Doerks T, Stark M, Muller J, Bork P, Jensen LJ, von Mering C. The STRING database in 2011: functional interaction networks of proteins, globally integrated and scored. Nucleic Acids Res. 2011; 39:D561-68.

https://doi.org/10.1093/nar/gkq973

PMID:21045058

27. Sidis $Y$, Schneyer AL, Keutmann HT. Heparin and activin-binding determinants in follistatin and FSTL3. Endocrinology. 2005; 146:130-36.

https://doi.org/10.1210/en.2004-1041 PMID: 15471966

28. Ding $T, H e X Z, X u X L, X u H Y$, Zhou $C X$, Wang YJ. [Expression of serum FSTL-1 in bone metastasis of prostate cancer and its clinical implication]. Zhonghua Nan Ke Xue. 2014; 20:1090-92.

PMID:25597175

29. Peng $X$, Wang $P$, Li $S$, Jiang $Y$, Wu C. Follistatin-like protein 1 knockdown elicits human gastric cancer cell apoptosis via a STAT6-dependent pathway. Oncol Rep. 2019; 42:2806-13. https://doi.org/10.3892/or.2019.7334 PMID:31578589
30. Li C, Dai L, Zhang J, Zhang $Y$, Lin $Y$, Cheng L, Tian $H$, Zhang $X$, Wang $Q$, Yang $Q$, Wang $Y$, Shi G, Cheng F, et al. Follistatin-like protein 5 inhibits hepatocellular carcinoma progression by inducing caspase-dependent apoptosis and regulating Bcl-2 family proteins. J Cell Mol Med. 2018; 22:6190-201. https://doi.org/10.1111/jcmm.13906 PMID:30255547

31. Fabian MR, Sonenberg N, Filipowicz W. Regulation of mRNA translation and stability by microRNAs. Annu Rev Biochem. 2010; 79:351-79. https://doi.org/10.1146/annurev-biochem-060308103103 PMID:20533884

32. Gulyaeva LF, Kushlinskiy NE. Regulatory mechanisms of microRNA expression. J Transl Med. 2016; 14:143. https://doi.org/10.1186/s12967-016-0893-x PMID:27197967

33. Zhang $Y$, Fu J, Zhang Z, Qin H. miR-486-5p regulates the migration and invasion of colorectal cancer cells through targeting PIK3R1. Oncol Lett. 2018; 15:7243-48. https://doi.org/10.3892/ol.2018.8233 PMID:29725442

34. Chen $H$, Ren $C$, Han C, Wang D, Chen $Y$, Fu D. Expression and prognostic value of miR-486-5p in patients with gastric adenocarcinoma. PLoS One. 2015; 10:e0119384.

https://doi.org/10.1371/journal.pone.0119384 PMID:25793394

35. Youness RA, El-Tayebi HM, Assal RA, Hosny K, Esmat G, Abdelaziz Al. MicroRNA-486-5p enhances hepatocellular carcinoma tumor suppression through repression of IGF-1R and its downstream mTOR, STAT3 and c-Myc. Oncol Lett. 2016; 12:2567-73. https://doi.org/10.3892/ol.2016.4914 PMID:27698829

36. Ren C, Chen H, Han C, Fu D, Zhou L, Jin G, Wang F, Wang $D$, Chen $Y$, Ma L, Zheng X, Han D. miR-486-5p expression pattern in esophageal squamous cell carcinoma, gastric cancer and its prognostic value. Oncotarget. 2016; 7:15840-53. https://doi.org/10.18632/oncotarget.7417 PMID:26895105 


\section{SUPPLEMENTARY MATERIALS}

\section{Supplementary Figures}
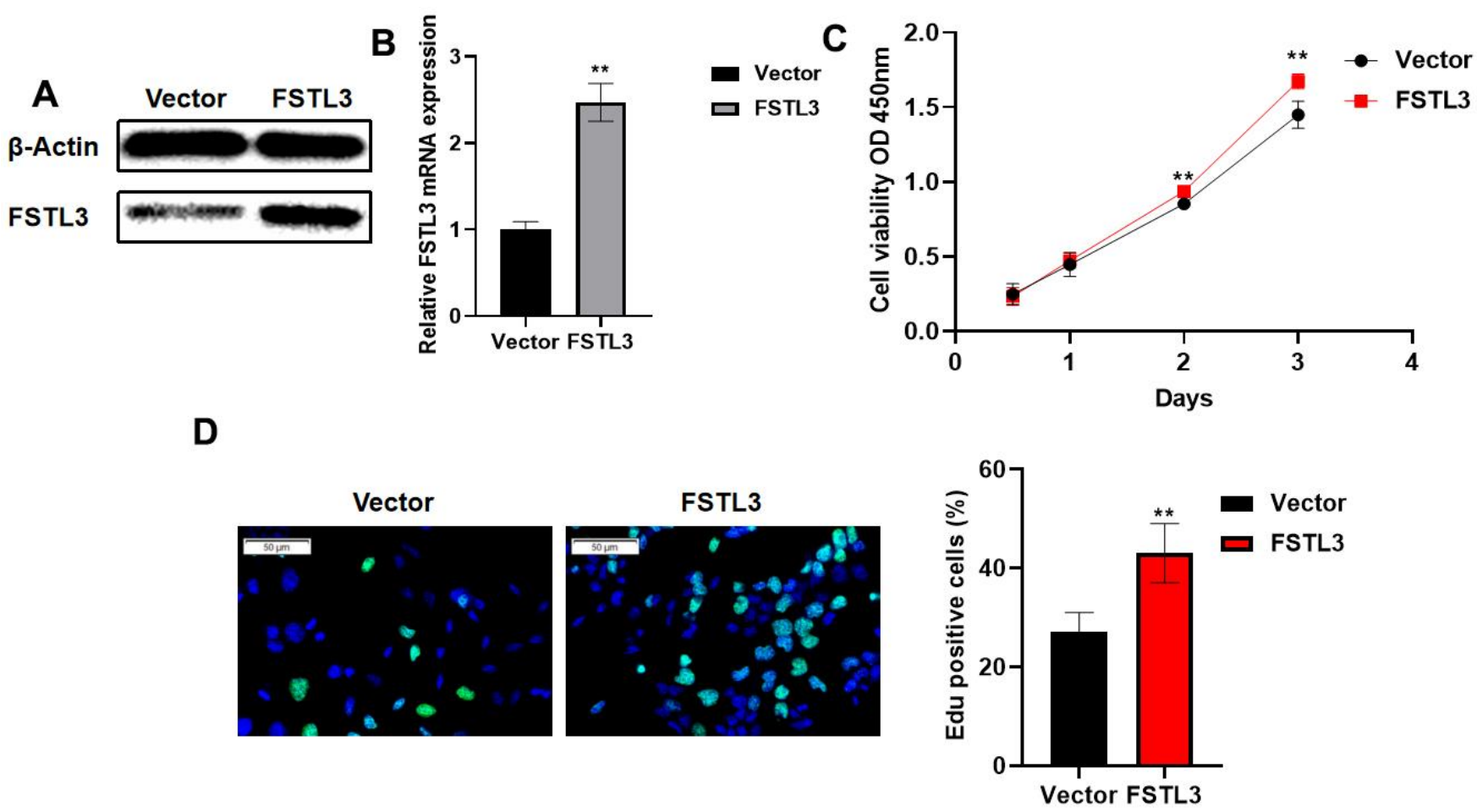

E

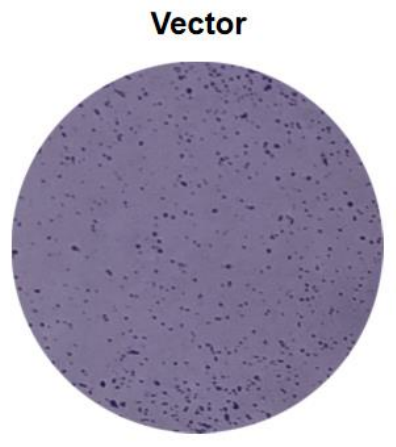

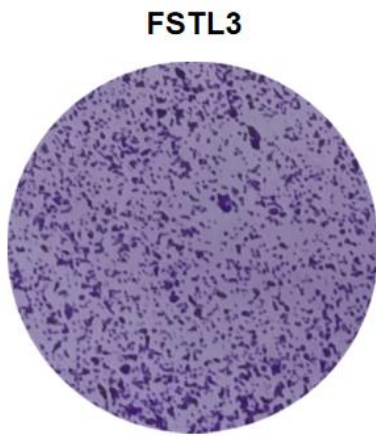

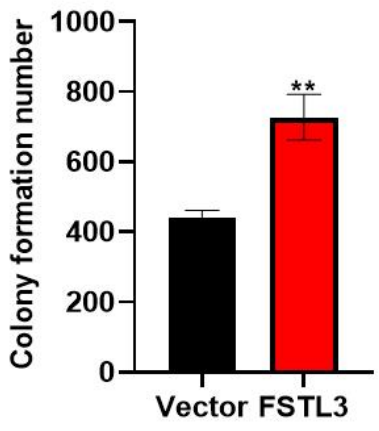

Vector

口 FSTL3

Supplementary Figure 1. FSTL3 overexpression promotes GES1 cell viability and proliferation. (A) Overexpression of FSTL3 in GES1 cells monitored using Western blotting; monitoring the housekeeping protein $\beta$-actin was used as a loading control. (B) Overexpression of FSTL3 in GES1 cells monitored using qRT-PCR. (C) Overexpression of FSTL3 in GES1 cells assessed by using the cell viability assay. (D) FSTL3 overexpression in GES1 cells was assessed for effects on cell proliferation using EdU incorporation assay. Cell proliferation was determined by EdU staining. (E) FSTL3 overexpression and effects on MGC-803 cell colony formation. Error bars indicate \pm SEM; significance indicated by asterisks, ${ }^{*} P<0.05,{ }^{*} P<0.01, * * * P<0.001$; number of experiments, $\mathrm{n}=3$. 

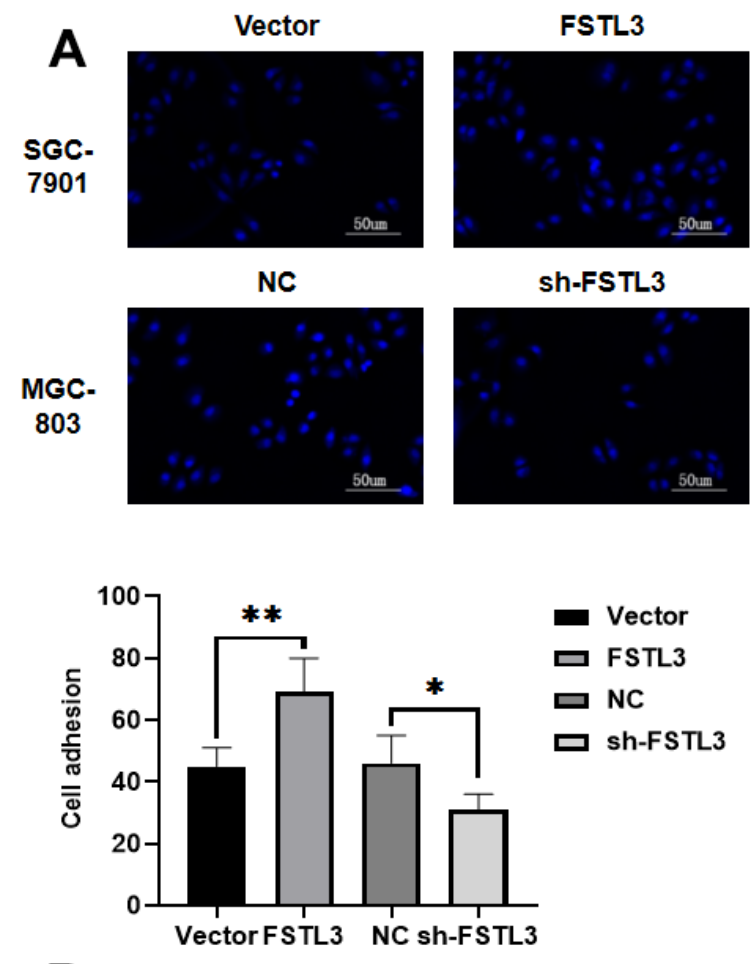

B

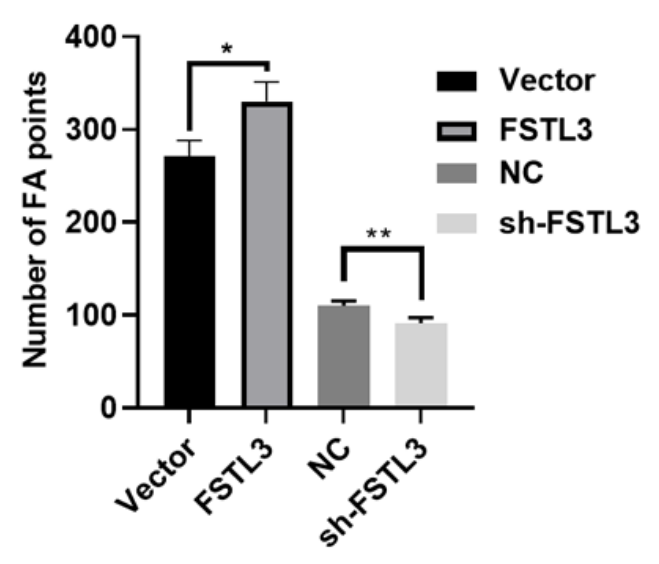

Supplementary Figure 2. FSTL3 overexpression promotes cell adhesion activity and the number of focal adhesion. (A) FSTL3 overexpression in MGC-803 cells was assessed for effects on cell adhesion ability. The adhesion ability of MGC-803 cells is expressed as an adhesion ratio. (B) FSTL3 overexpression and effects on the number of MGC-803 cell adhesion spots. Error bars indicate +SEM; significance indicated by asterisks, ${ }^{*} \mathrm{P}<0.05,{ }^{*} \mathrm{P}<0.01,{ }^{* * *} \mathrm{P}<0.001$; number of experiments, $\mathrm{n}=3$. 